\title{
Experimentation on the Concentrator Tree with Loopback
}

\author{
William S. Hiles and David T. Marlow \\ Systems Research and Technologies Department \\ Combat Systems Branch \\ Naval Surface Warfare Center, Dahlgren Division \\ Dahlgren, Virginia 22448-5000
}

\begin{abstract}
The FDDI concentrator tree topology with loopback has been proposed for use in networks which need to continue operation after multiple faults. The initial experiments to use the concentrator tree with loopback resulted in configurational dependencies which were not initially understood. A few caveats have been found which can be handled through careful cabling. This document is intended to provided both a theoretical and experimental analysis of various cases of the concentrator tree with loopback. In addition, a discussion on the use of bypass relays in the concentrator tree with loopback is provided.
\end{abstract}

\section{Introduction}

The Fiber Distributed Data Interface (FDDI) family of standards provides specifications which support many different approaches to configuring a network[3]. In some environments, events may occur which lead to multiple faults occuring. This can cause isolation of stations referred to as segmentation. The resulting independent islands of operational equipment may not be able to perform their purpose. Segmentation is considered significant when two or more systems are separated from two or more other systems on independent islands in a network. A network may be made more survivable. This may be done by using a configuration approach that reduces link vunerability and/or using redundant resources.

The FDDI is an extremely robust local area network due to its fault tolerance capabilities which have been designed into the core of the standard. Under the basic interconnection schemes, it is straight forward to design a configuration approach that can survive one link failure. . However, the robustness of FDDI provides a unique opporturnity to achieve higher degrees of survivability using creative topologies.

\section{Concentrator Tree}

A concentrator tree is a method of interconnecting concentrators such that the A and B ports of each concentrator in the tree connect to $M$ ports of the same concentrator higher in the tree as shown in Figure 1. The Concentrator Tree has been discussed in the SAFENET [1] standardization committee within the Navy where there is interest in continued operation after multiple cable faults. This con-
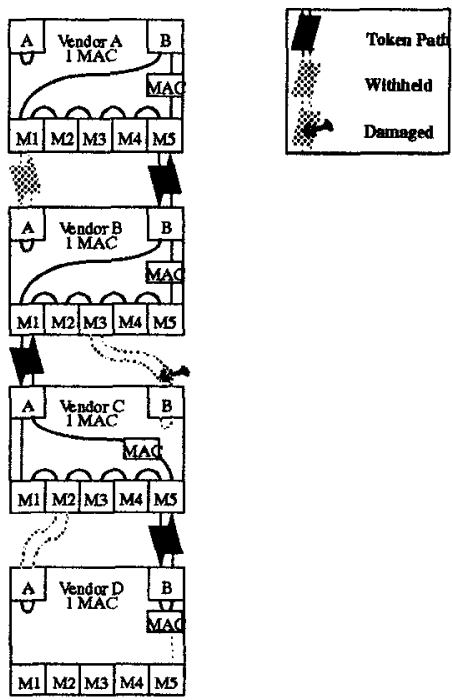

Figure 1: Concentrator tree

figuration is the basis for a reconfiguration technique being considered at Martin Marietta(Moorestown NJ) named "Alternate Path FDDI"[2]. The configuration shown in Figure 1 utilizes the mechanisms necessary for dual-homing[3] in order to provide redundant backup links and requires only single MAC concentrators. In SMT 7.2 [4], the dual-homing capability is allowed in section 5.2.3.

This section of the FDDI standard allows the tree to be configured such that there is a redundant set of connections within the tree but does require that the backup connection be used only as a result of the loss of the active token path as indicated in the third sentence of section 5.2.3.

As indicated in Figure 1, the token path is routed through the B port of each concentrator as long as the port is available. For future reference, the $M$ ports have been numbered M1 through M5, and the concentrators are identified as Vendor A through Vendor D. When the ring is broken as is shown between Vendor B and Vendor C, the token path cannot go through the B, port and the Vendor $\mathrm{C}$ concentrator allows the $\mathrm{A}$ port to become the active port of the concentrator. This hopping mechanism is the desired operation of the topology. The tree configuration guarantees that the token path be routed through the MAC 
in the station with the definition in section 5.1 of the SMT 7.1 specification:

"...If it [the concentrator] has at least one MAC(s), a MAC shall be located at the exit Port(s) (i.e., a MAC follows the stations inserted on its $\mathrm{M}$ ports)..."

The operation of the concentrator tree is very simple and utilizes essentially the same mechanisms as dualhoming. When the network is functioning normally with no damage, the A ports of each concentrator will be withheld. In the event that a fault occurs on an active linked pair (with reference to Figure 1, on the B port between Vendor $\mathrm{B}$ and Vendor $\mathrm{C}$ ), the concentrator below the fault will switch to the A port and use it as the active link. In the event that the fault on the B port is corrected, the concentrator will withhold the A port and switch back to the $B$ port. This operation occurs on the order of a few milliseconds for most equipment but such timing is implementation/vendor specific.

The concentrator tree alone is no more survivable than approaches such as dual-homing because damage to any set of links still results in isolation. When the links on both the $\mathrm{A}$ and $\mathrm{B}$ ports of a concentrator (for example, between Vendor $B$ and Vendor $C$ ) within the tree are broken, the tree will form two segmented trees. Thus segmentation can be caused by as few as two faults in the concentrator tree of Figure 1. As previously described, such segmentation may result in system degradation and even failure due to the islands of equipment being separated and unable to communicate.

\subsection{Concentrator Tree with Loopback}

Mechanisms which can survive more than two link failures are of interest to some applications. Use of Network layer protocols [3] on top of FDDI can provide any level of redundancy required; however, such solutions are typically slow in switching to a surviving data path. One technique [2] proposed involved using a concentrator tree with connections between the top and bottom of the tree. This configuration is called the concentrator tree with loopback and is illustrated in Figure 2.

Initial experiments with this configuation produced mixed results. Problems with segmentation occurred in some configurations but not in others. Theoretical work and experimentation were needed to identify and resolve these problems.

The concentrator tree with loopback creates two rings. The first ring (the one that exits the B port) is shown as a hatched path and the second ring (the ring which enters the B port) is shown as a solid path. Note that only the hatched ring connects the concentrator MACs and the

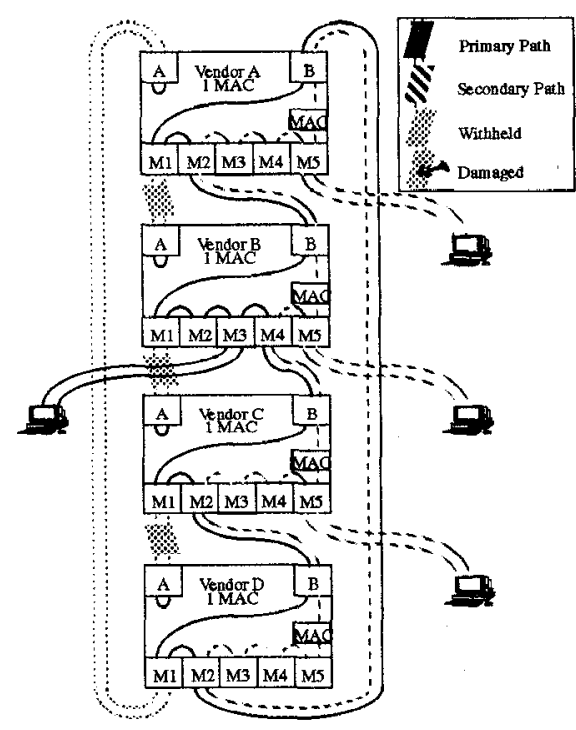

Figure 2: Undamaged concentrator tree with incorrect loopback

user stations on the right side of Figure 2. The solid path also maintains a token due to the station shown on the left of Figure 2. In order to avoid segmentation, the MACs of all stations including the concentrators must be on the same ring both before and after reconfiguration. Maintaining all MACs of the network connected on the same ring is referred to as "correct loopback" in this paper. By looping back to the top, this configuration prevents isolation from happening and essentially requires at least four breaks to cause segmentation. For correct operation there is actually no need for a token to be circulated on the second path. When additional stations are added to the concentrator M ports, it is possible for some of the stations to be divided between two separate token paths. This is illustrated in Figure 2 and is referred to as incorrect loopback. The station on the left of the concentrator tree cannot communicate with the other stations and it cannot communicate with any of the concentrators.

Since the rings do not wrap at any point in Figure 2, any station which connects to an $\mathbf{M}$ port on the solid path will have a completely separate token from the stations on the hatched path. Without loopback the concentrator tree relies on the wrap points at the top and the bottom of the concentrator tree to force all the $M$ ports to be included in a single token path. When the loop is formed, once the token leaves a concentrator to enter another concentrator, it will not return through the $M$ port and all successive $M$ ports are bypassed.

This segmentation problem can be clarified as follows. When additional stations are connected to the concentrators using $\mathrm{M}$ ports between the $\mathrm{A}$ and $\mathrm{B}$ port connections of the succeeding concentrator, the loops will cause the $M$ 
ports to be separated to a different data path than the MACs of the concentrators. Further, if the connections are arbitrarily chosen, any station connected between the A/B to $\mathrm{M}$ connections may be isolated from the stations connected after the A/B connections. After reconfiguration, stations will then change the location within the concentrator paths and may move from one token path to the other. The concentrator's MAC has no knowledge of the existence of the MACs connected to the other data path; and, equipment on each path cannot communicate.

This problem can easily be overcome through careful interconnection utilizing the following rules.

1. The A port of a succeeding concentrator must be connected to the first $\mathrm{M}$ port of the physical token path.

2. The B port of a succeeding concentrator must be connected to the second $M$ port of the physical token path.

Additional $\mathrm{M}$ ports (beyond the first two) can be added to the concentrators starting at the third $\mathrm{M}$ port in the physical token path as shown in Figure 3. In order for the loopback configuration to operate correctly without bypassing $\mathrm{M}$ ports during reconfiguration, the internal connection of the concentrator must be known and the $\mathrm{A}$ and $B$ ports of the successive concentrators must be connected using these rules. The FDDI standard does not dictate the order of the $\mathrm{M}$ ports in the token path of a concentrator but provides indexing for management purposes. In some concentrators the indexing of the $\mathrm{M}$ ports is not necessarily the order in which the physical data path will flow; thus, the loopback configuration requires prior knowledge of the architecture of the concentrator to determine the first and second $M$ ports in the physical token path. This information should be obtained prior to purchasing a concentrator to be used in the loopback configuration.

Figure 4 is provided to illustrate the operation of the concentrator tree with correct loopback. In this figure, the $B$ port connection of Vendor $C$ becomes inactive which enables the A port. This in turn routes the token path through the A port of Vendor $C$ from the M1 port of Vendor B without affecting any other stations in the network. Further, two other connections within the network may be damaged without any chance of segmentation as shown in the breaks between Vendor $A$ and Vendor $B$. This provides better reconfiguration capabilities than can be achieved on either the trunk ring or through dual-homing alone. Note that all stations in Figure 4 remain connected on the same data path regardless of damage done to the links between the concentrators.

If the previously stated rules are not followed, stations may be attached to the inactive data path after damage oc-

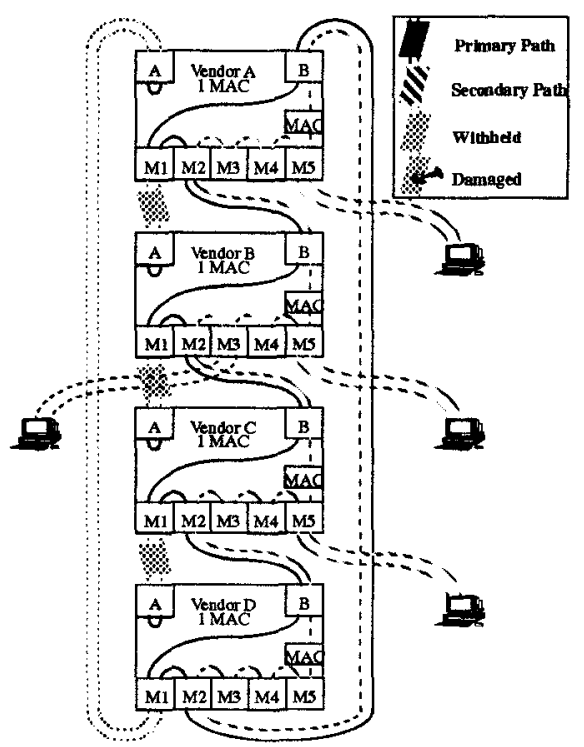

Figure 3: Undamaged concentrator tree with correct loopback

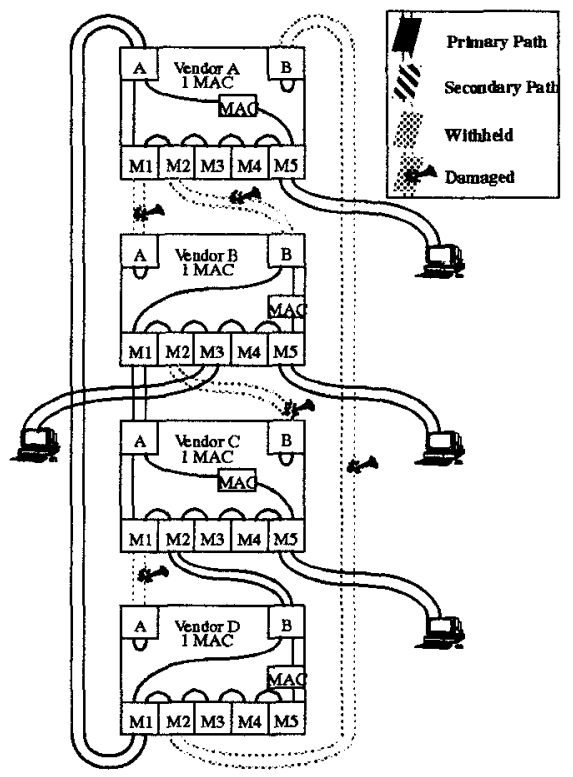

Figure 4: Damaged concentrator tree with correct loopback

curs on the network. Since this path is a usable path, the station will be added to the ring and the station will initiate a token. Two separate tokens will then flow in the network and the stations on separate paths will not be interconnected. Figure 5 illustrates this via a damaged link to the configuration of Figure 2. The stations connected to the concentrators with the Solid lines are stations on the primary data ring and the stations connected with hashed lines are on the secondary data path. Note in Figure 5 that 
the station on the left of the concentrators would have been on the primary data ring (the solid ring) but after this damage, the station is moved to the secondary data ring (the hashed ring). The station on the bottom right will always connect to the secondary data ring unless the link between the Vendor $\mathrm{C}$ concentrator and the $\mathrm{B}$ port of Vendor $\mathrm{D}$ concentrator is broken.

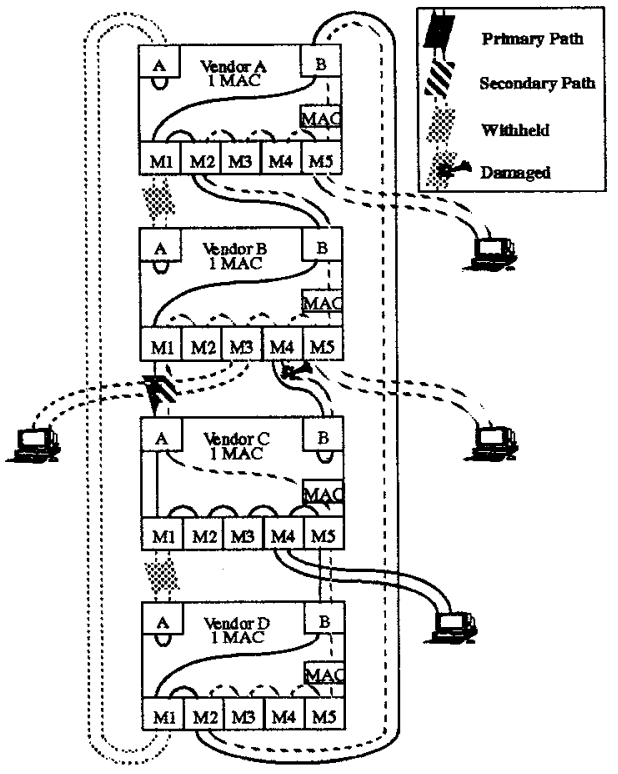

Figure 5: Concentrator tree with incorrect loopback

Note that there are no capabilities built into FDDI which will identify that a concentrator tree with incorrect loopback has been configured. A system may be setup up correctly and at some point later in the life of the system it may be cabled incorrectly. This presents a significant problem for system management and maintenance.

\subsection{Optical Bypasses for the Concentrator Tree with Loopback}

Operation of the concentrator tree with loopback cannot be maintained when more than one concentrator is powered down. To overcome this problem, optical bypasses can be added which allow concentrators to be powered down without causing isolation of concentrators or stations connected through other concentrators as shown in Figure 6. There are drawbacks to this type of solution. Typical commercially available optical bypasses consume a great deal of power from the optical signal, and this limits the number of consecutive stations which can be powered down. Testing of standard optical bypasses has shown that no more than two stations can be bypassed using FDDI power levels and current commercially available optical bypasses. Bypassing more stations pushes the power limits for the stations. This results in spurious er- rors due to the reduced signal to noise ratio $s$ the active FDDI links to reset.

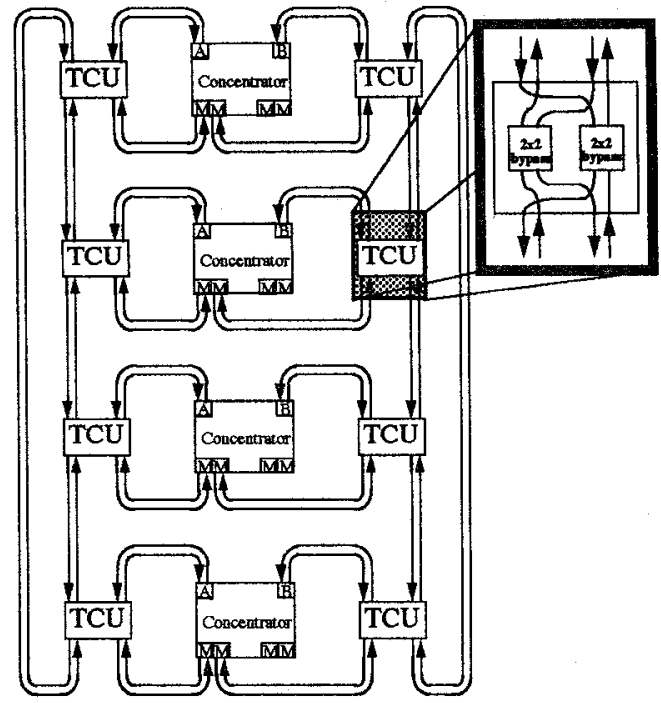

Figure 6: Concentrator Tree with Optical Bypasses

\section{Basic System Configuration for Testing}

Figure 6 shows the basic system configuration that will be referred to as the Concentrator Tree with Loopback. The basic assumptions for this configuration are that each active FDDI device in the network is a concentrator containing at least one MAC entity. Optical bypasses (as shown in Figure 6) may be used to enahnce the operation of the network by allowing more stations to be powered down without causing segmentation. The concentrators in this configuration may function as both a concentrator and a station meaning that the device may contain a full protocol stack and a suite of applications. Further, the concentrators may contain additional $M$ ports to allow for additional stations to be attached to the network.

\subsection{Testable System}

Unfortunately, our Data Technologies Lab does not contain enough equipment to test the exact configuration shown in Figure 6. Compromises must be made in order to test the operation of the network. The first compromise is that none of the concentrators that were available for test had the capability to contain a full protocol stack. Although not necessary for operation, it is important for at least one of the concentrators to be accessible through a workstation to determine the state of the network during the testing procedure. To provide this facility, stations were attached to the concentrators through the additional $\mathrm{M}$ ports on the concentrators. Figure 7 shows the same configuration as Figure 6 without the optical bypasses. 


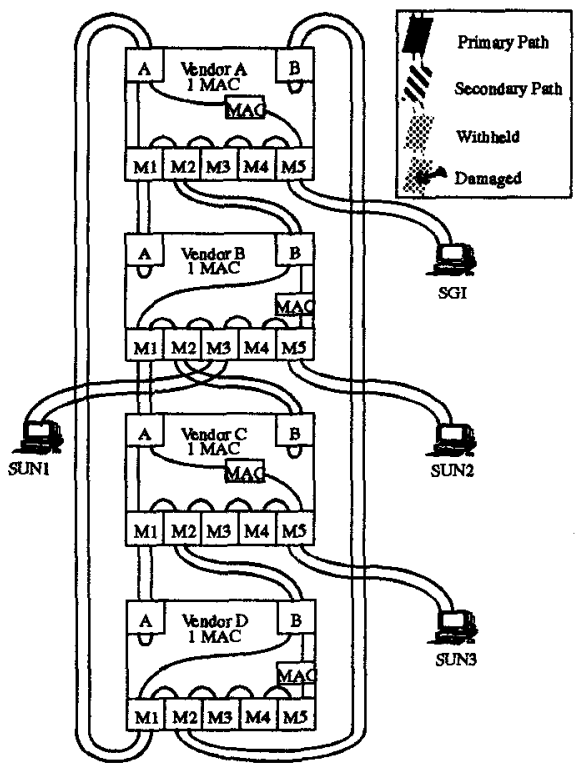

Figure 7: Concentrator tree with correct loopback

\subsection{Testing Tools}

The concentrators which were available for testing the concentrator tree are listed in Table 1. Likewise, Tables 2 and 3 show the available stations and software for monitoring and measuring the test.

Table 1: Available Concentrators

\begin{tabular}{|l|l|c|c|c|}
\hline \multicolumn{1}{|c|}{ Vendor } & Model & $\begin{array}{c}\# \\
\text { MAC }\end{array}$ & $\begin{array}{c}\text { M } \\
\text { ports }\end{array}$ & SMT \\
\hline Synernetics & $\begin{array}{l}\text { LanPlex } \\
5004\end{array}$ & 2 & 8 & 6.2 \\
\hline NPI & $695-0103$ & 1 & 6 & 7.2 \\
\hline NPI & $695-0103$ & 1 & 6 & 6.2 \\
\hline Synoptics & 3000 & 3 & 8 & 6.2 \\
\hline
\end{tabular}

Table 2: Available Monitoring Stations

\begin{tabular}{|l|l|c|c|}
\hline Vendor & \multicolumn{1}{|c|}{ Model } & Ports & SMT \\
\hline Sun & $4 / 370$ & A/B & $6.2(\operatorname{Rev} 2.0)$ \\
\hline Sun & DTII & A/B & $6.2(\operatorname{Rev} 2.0)$ \\
\hline Sun & IPX & S & $6.2(\operatorname{Rev~1.0)~}$ \\
\hline SMI & Iris 4D/35 & A/B & 6.2 \\
\hline DTI & LanHawk & - & - \\
\hline
\end{tabular}

Of the concentrators available for testing, the Synernetics LanPlex 5004 and the Synoptics 3000 provide the greatest flexibility. These concentrators contains three internal data paths which are configured as a primary, secondary, and local. The A and B ports are always
Table 3: Available Network Management Software

\begin{tabular}{|l|l|c|c|c|}
\hline \multicolumn{1}{|c|}{ Vendor } & Name & Ver & SMT & SNMP \\
\hline Sun & SNM & 2.0 & N & Y \\
\hline SGI & FDDIVis & 3.0 & Y & N \\
\hline Synernetics & Viewplex & 2.0 & Y & Y \\
\hline Synoptics & Optivity & 3.0 & Y & Y \\
\hline
\end{tabular}

connected to the primary and secondary paths. Any of the M ports may be selectively moved to any of the three paths. The Synernetics concentrator contains a "roving" MAC which may be moved to any of the paths. By moving the second MAC to the local path, the concentrator can be configured as a single MAC concentrator. Similarly, the Synoptics concentrator contains a roving MAC which serves the same purpose; however, the Synoptics concentrator also contains a third MAC which is fixed to the secondary ring.

Having two Network Peripherals Inc. (NPI) concentrators with identical hardware and different versions of SMT provided additional capabilities for hardware testing. One of the concentrators has been held at revision 6.2 so that any odd events found in the 7.2 version of SMT may be traced and identified. The NPI concentrators are less expensive and provide a single MAC which is always associated with the primary ring as defined in the FDDI specification. Additionally, any of the M ports on the NPI concentrators may be enabled or disabled selectively through a console attached to the concentrator.

In addition to the five concentrators, a DTI LanHawk FDDI analyzer was available for tracking down problems. This piece of equipment is a transparent analyzer that connects to the network through an optical splitter. With this piece of hardware it is possible to monitor the data that is on the network transparently (i.e. without interrupting the data or network configuration since it does not act as a source on the ring). The only effect that the LanHawk has on the network is a degradation of the optical power between the stations in which the analyzer is connected as a result of connecting the device through a optical splitter. The DTI analyzer used in the testing has a single passive network tap so it is only possible to monitor one ring of the network at a time

The most useful software was the FDDI Visualizer distributed by SGI for the Silicon Graphics machines. The advantage of this software is that it provides a "SMT only" means of viewing the network and also is quicker to recognize changes in the network than the SNMP [6] based tools. The Viewplex software by Synemetics is unique in that it provides the capability to quickly examine the complete SMT MIB of any station which is recognizable on the network. Optivity was provided by Synoptics and provides very similar capabilities as View- 
plex. The combination of these products provided a thorough view of the internal state of the concentrators on the network.

\subsection{Testing Goals}

One goal of this paper is to show the validation of the rules described in section 2.1 by providing the results of testing using configurations which do and do not conform to these rules. This experiment was initially carried out to determine if there was any data maintained by the concentrators which will allow them to determine if the network had been incorrectly configured. A second goal of the experimentation was to validate the operation of the concentrator tree with loopback under various damage scenarios for components, bypasses, and fibers. A final goal of the experimentation was to verify that the operation of the concentrator tree with loopback is not dependent on the ordering of the concentrators. Each test was performed multiple times with the concentrators in different orders.

\section{$4 \quad$ Validation of Rules}

\subsection{Concentrator Tree Without Loopback}

Before starting the tests, the basic operation of the concentrator tree without loopback had to be established. This provided a basis to determine if the loopback actually changed the ability of the network to function. Figure 8 illustrates the concentrator tree without loopback in which the station at the top forms a wrap point at the B port and the station at the bottom forms a wrap at the M ports. As a result, the token flows on the data paths as would normally occur in a tree configuration.

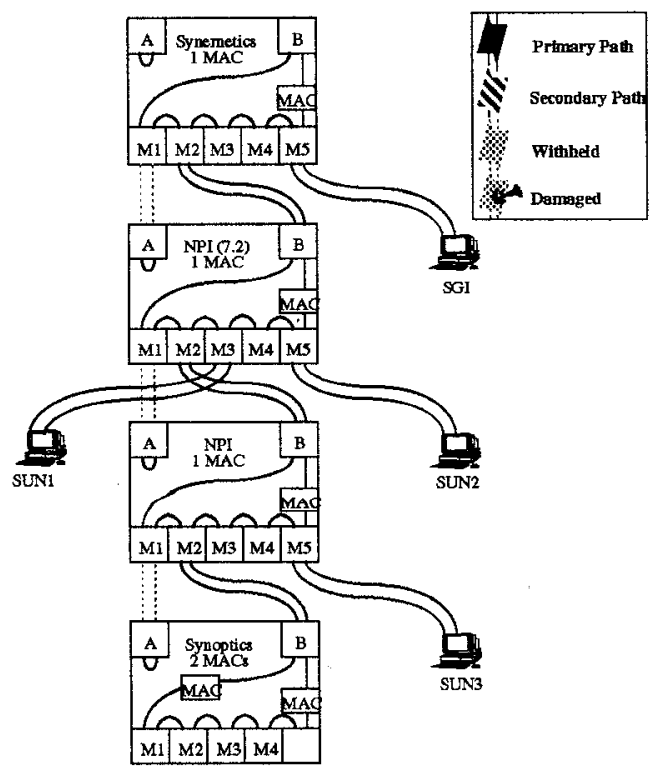

Figure 8: Undamaged concentrator tree without loopback
When this network is in operation, the states of the concentrators are listed in Table 4. Note that the NPI concentrators with SMT 6.2 was reported as being in the Thru mode by Viewplex. Viewplex attempted to extrapolate the status of the NPI concentrator which does not contain SNMP (i.e. the one with version 6.2 of SMT) and reported a Thru state. Additionally, Viewplex also reported that the Synoptics concentrator was in a Thru state when the front panel and Optivity indicated that it was in a WrapAB state. Viewplex was easily confused when the state of the network changed as the concentrators switched from one port to the other. Viewplex dropped the ring and indicated an error. After a significant period of time, Viewplex was able to rebuild its interpretation of the network. Once Viewplex stabalized on a configuration, it showed the stations on the trunk ring in one view and the stations in the tree in another view.

Table 4: State of Concentrators for Normal Concentrator Tree as reported by Viewplex

\begin{tabular}{|c|l|l|l|l|}
\hline$\#$ & \multicolumn{1}{|c|}{ Vendor } & \multicolumn{1}{|c|}{ State } & Fault & Monitor \\
\hline 1 & Synernetics & Isolated & none & VP $^{\mathbf{a}}$ \\
\hline 2 & NPI (7.2) & WrapB & none & FV $^{\mathbf{b}}$ \\
\hline 3 & NPI (6.2) & Thru & none & \\
\hline 4 & Synoptics & WrapAB & none & $\mathrm{OPt}^{\mathbf{c}}$ \\
\hline
\end{tabular}

a. ViewPlex by Synernetics

b. FDDIVisualizer by Silicon Graphics Inc.

c. Optivity by Synoptics

Optivity showed promise because it did not incorrectly identify the states of the NPI concentrator; rather, it simply refused to show the NPI with 6.2. in the network. Optivity did not distinguish between stations in the tree and showed the logical token path. As a result, it was not easy to identify which concentrator was at the head of the tree based on the display.

When this portion of the experimentation started, it was thought that the network management tools would be of great help in identifying what was actually going on within the network. However, as a result of the problems associated with the tools, it became evident that the only truly reliable means for identifying the state of the concentrators was to physically examine the status displayed on the panels of the concentrator or to connect RS232 terminal to the service port and watch for state indications.

The network management software was used for the later experiments, but it was used only to examine the MIB of the individual concentrators. In general, this experimentation needed a display of the token path, and that was adequately handled by the FDDI Visualizer which was not without its own problems. 


\subsection{Adhering to the Rules}

\subsubsection{Undamaged Case}

To test the operation of the concentrator tree with loopback, the network is setup adhering to the rules stated previously in section 2.1. This configuration is shown in Figure 3. Note that the primary MAC entity for each concentrator is located on the solid line indicating that they are interconnected. The hashed line indicates the second loop of the tree. The hashed ring contains a token presumably because the Synoptics concentrator has a MAC fixed to the secondary data path before the $\mathrm{M}$ ports in the concentrator. The existence of tokens on each ring was verified using the LanHawk analyzer.

Tests of this configuration using the FDDI Visualizer displayed all the stations (including the single attached stations) in a ring and displayed two tokens rotating around phantom rings in mid-screen. Breaking the ring to add a station to the tree confused the software, and it could not recreate the ring but still thought the phantom ring existed. The states of each of the concentrators (shown in Figure 3) is listed in Table 5 as determined by monitors on the service ports of the concentrators.

Table 5: Concentrator States for Undamaged Correctly Configured Loopback

\begin{tabular}{|c|l|l|l|l|}
\hline$\#$ & \multicolumn{1}{|c|}{ Vendor } & \multicolumn{1}{|c|}{ State } & Fault & Monitor \\
\hline 1 & Synernetics & WrapAB & & VP \\
\hline 2 & NPI (7.2) & Wrap B & & FV \\
\hline 3 & NPI & Wrap B & & \\
\hline 4 & Synoptics & WrapAB & & OPt \\
\hline
\end{tabular}

The information on the FDDIVisualizer does show enough information to determine which stations are visible on the same ring as the SGI station. The information for the remaining tests only requires that the stations can be reached for the various configurations. This will indicate if the MAC of the station is accessible on the different token paths.

\subsubsection{Damaged Case}

To start the tests for the case with a damaged concentrator tree, the network is set up such that it conforms to the rules in section 2.1. After setting up the network, regardless of the damage (other than damage causing segmentation) all stations were visible from the FDDI Visualizer. Figure 9 shows the configuration of the damaged tree. Regardless of which of the vacant $\mathbf{M}$ ports were used, the stations were always visible under any of the fault sceneries. Further testing proved that the ordering of the concentrators did not affect the test.

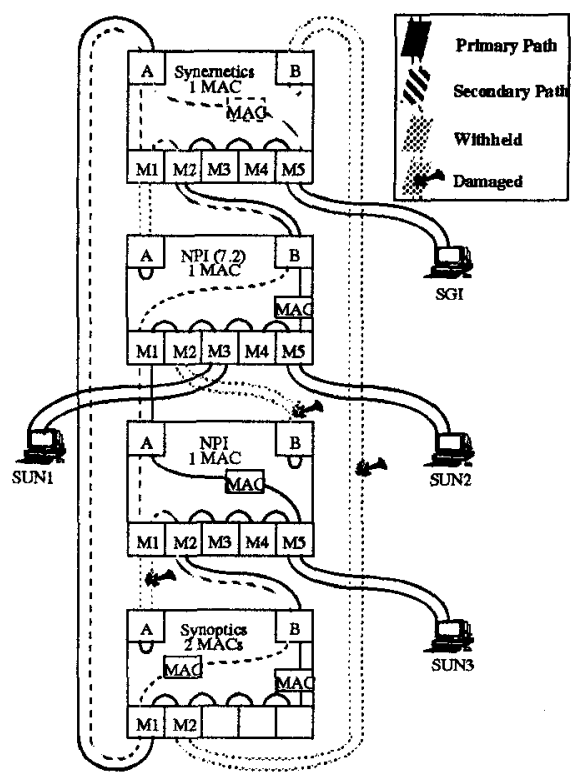

Figure 9: Damaged concentrator tree with correct loopback

\subsection{Violating the Rules}

\subsubsection{Undamaged Case}

To test the rules, the concentrators were set up exactly the same as in the previous test except that the connection to the B port of the NPI (6.2) was connected to the fourth port of the NPI (7.2) concentrator as shown in Figure 10. In this configuration, the machine labeled "sun1" was connected to the third $\mathrm{M}$ port as in the previous test. When viewing the stations through the FDDI Visualizer, the station labeled sunl does not appear to be addressable.

After connecting the SGI machine to the second port of the NPI (7.2) concentrator, the FDDI Visualizer indicated that the only two stations on the ring are the SGI and the station labeled sun1. This configuration is shown in Figure 11 .

This validates that the two separate token paths exist. For each concentrator, the ports were switched such that the $\mathrm{M}$ port with the monitoring station attached was before, between and after the tree links that form the main loops. The results from each tested configuration validated the rules in section 2.1 .

\subsubsection{Damaged Case}

If the concentrator tree shown in Figure 10 is damaged such that the link at the B port of the NPI concentrator is broken, the NPI concentrator will switch to the A port. When this occurs, all the $\mathrm{M}$ ports are to the right of the active $\mathrm{M}$ to A connection between NPI (6.2) and NPI (7.2). 


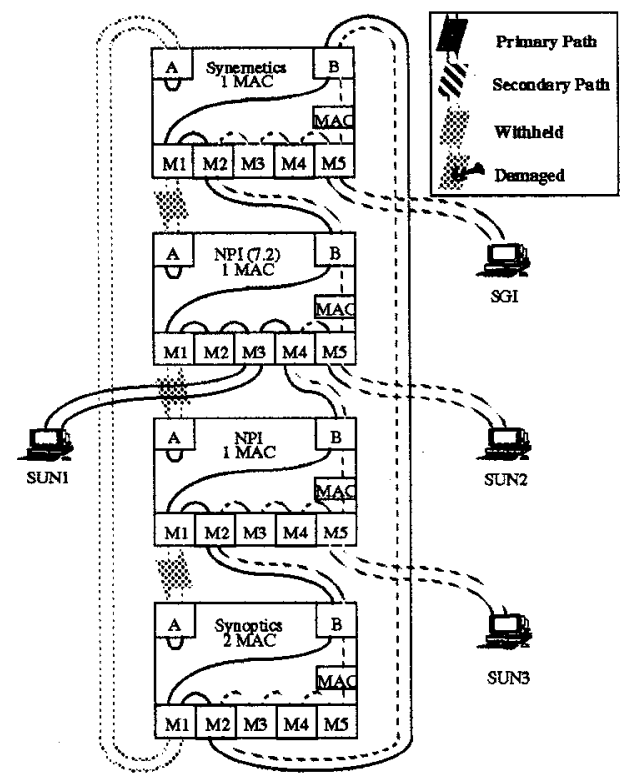

Figure 10: Undamaged concentrator tree with incorrect loopback (SGI on primary path)

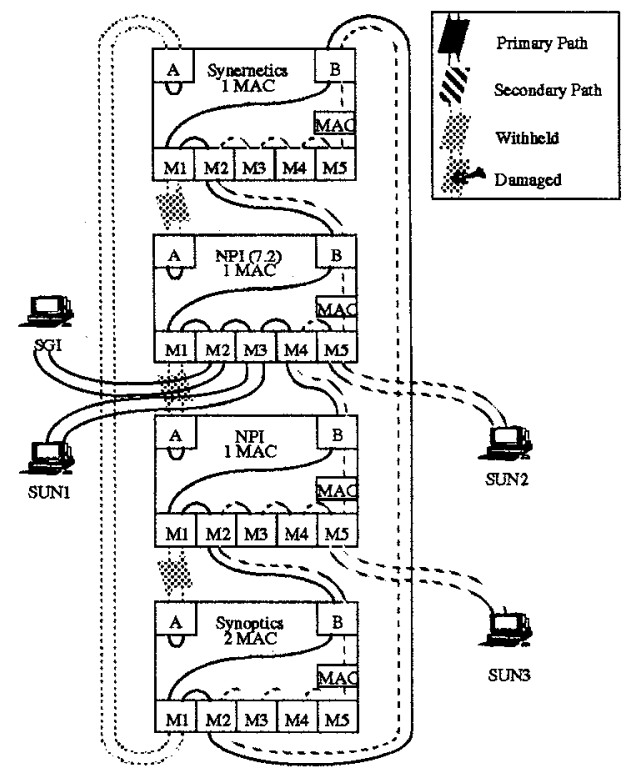

Figure 11: Undamaged concentrator tree with incorrect loopback (SGI on second path)

As a result, all the stations on the network move to the same ring as shown in Figure 12.

To test this scenario, the stations were set up as shown in Figure 12 and it was found that all stations existed on a single token path. This was validated by moving the monitoring station to the various $M$ ports on the concentrators. Whenever the monitoring station was set before the $M$

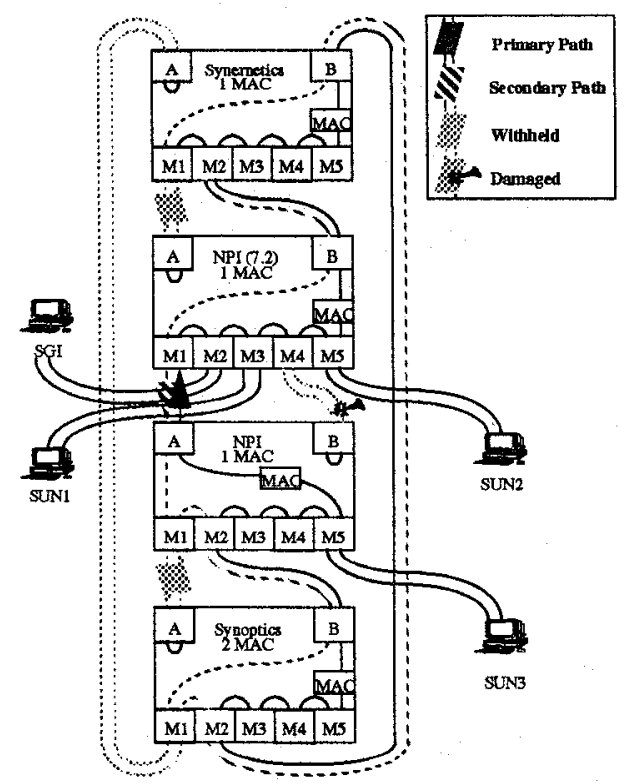

Figure 12: Damaged concentrator tree with incorrect loopback

ports between concentrators, it existed on a separate token path and none of the stations (including concentrators) could be seen. Upon moving back to a position after the connections between the concentrators the stations were again reachable.

\subsection{Rule Validity Conclusion}

Based on the results of these tests, the rules for the operation of the Concentrator Tree with Loopback described in section 2.1 appear to be correct. It follows that based on both the theoretical and experimental analysis of the concentrator, as long as the rules are followed, the concentrator tree will operate as expected.

\section{Optical Bypass Damage}

Optical bypasses may be used in the concentrator tree with loopback. The Optical Bypass for the concentrators in the Concentrator Tree with Loopback is possibly the only single point of failure for any part of the network. Even this point of failure results in minimal damage because it isolates a single station from the network but does not form islands of independent networks. There are two relevant cases for failure of the optical bypass: damage on the A port side, and damage on the B port side.

As described, the operation of the optical bypass shown in Figure 6 allows for a concentrator to be removed from the optical datapath without breaking the ring for an extended period of time. A side effect of this operation is that the bypass also connects the $\mathrm{M}$ port to the $\mathrm{A}$ or $\mathrm{B}$ port of the same concentrator when it is in the bypass mode. 
During normal operation the only time that the bypass would be in the bypass mode would be when the concentrator was powered down. However, in the event that damage occurs which disables the optical bypass, the $M$ port of the concentrator will become connected to the $A$ or B port of the concentrator and will form a valid connection.

\subsection{Damage on the A port side}

Damage to the optical bypass on the A side of the concentrator is relatively inconsequential unless the fibers to the $B$ port are damaged also. If the B port is active, the damage to the bypass on the A side will be undetectable because the A port is being withheld by the concentrator. Figure 13 shows the concentrator tree with damage to the A port side of the tree. The effective configuration of the network is shown in Figure 14.

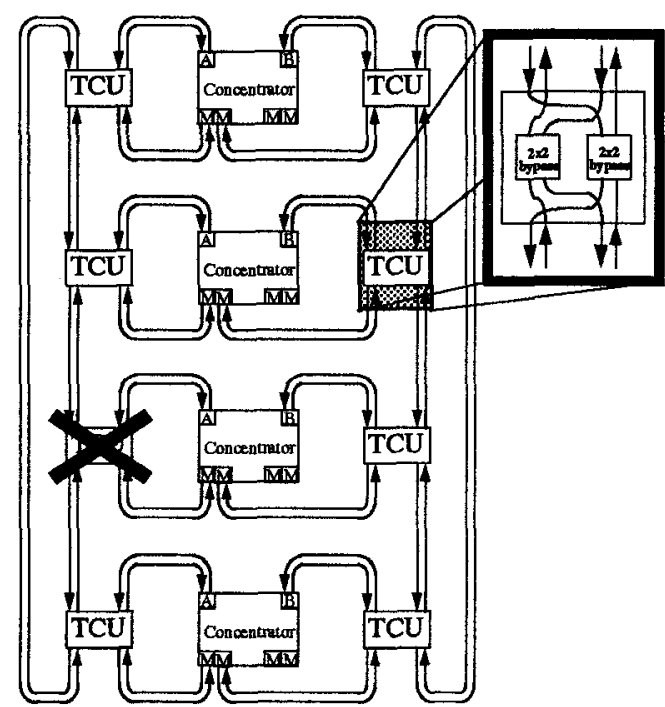

Figure 13: Concentrator Tree with Damaged Optical Bypasses on the A port

\subsection{Damage to the $B$ port side}

A more important case for consideration is when damage occurs to the bypass on the $B$ port side as shown in Figure 15. Figure 16 shows the effective configuration of the network after the optical bypass is damaged on the $B$ port side. Since the concentrator will switch to the A port only when the B port cannot pass a link confidence test, the concentrator will isolate itself. The B port will have a usable connection to its own $M$ port which will pass the link confidence test and so the A port will be withheld. The bypass will route the previous concentrator to the next concentrator so that the network is unaffected. This is effectively a single point of failure for the concentrator since it becomes isolated but operational after one failure.

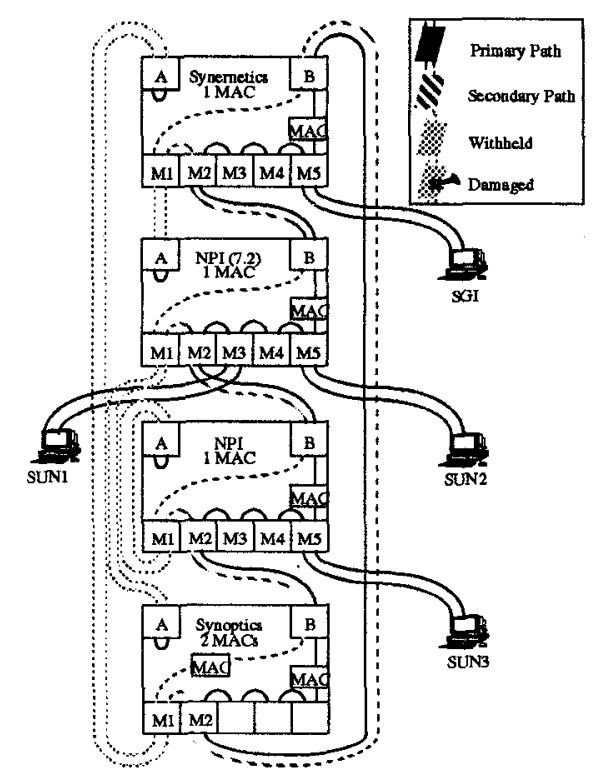

Figure 14: Effective Concentrator Tree with Correct Loopback and damage to an A port Optical Bypass

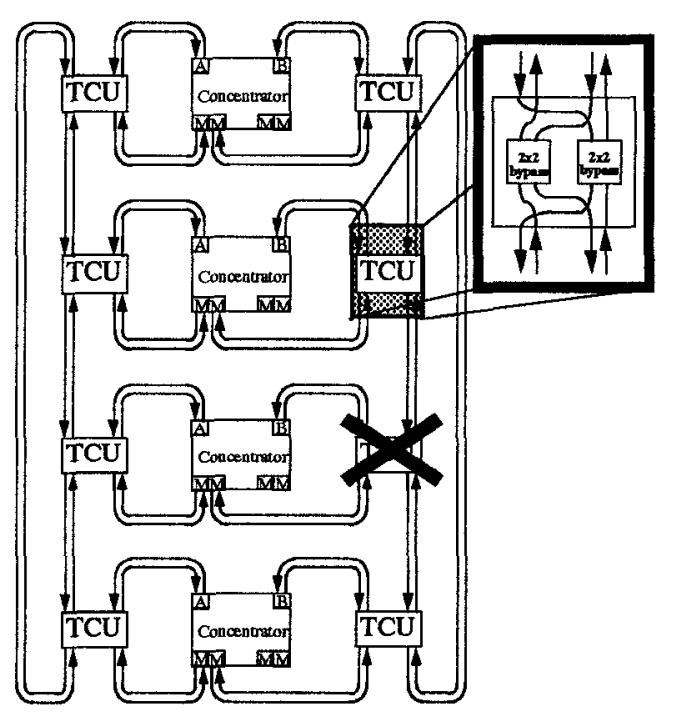

Figure 15: Concentrator Tree with Damaged Optical Bypasses

\subsection{A Different Bypass Configuration}

The optical bypass configuration used in Figures 13 and 15 shows the configuration from the SAFENET Handbook[7]. A different configuration can be created which will solve the problem of connecting the $M$ ports back to the B ports. Essentially, either optical switches or optical bypasses can be added to the bypass configuration so that when power is applied to the bypass unit, the station is in- 


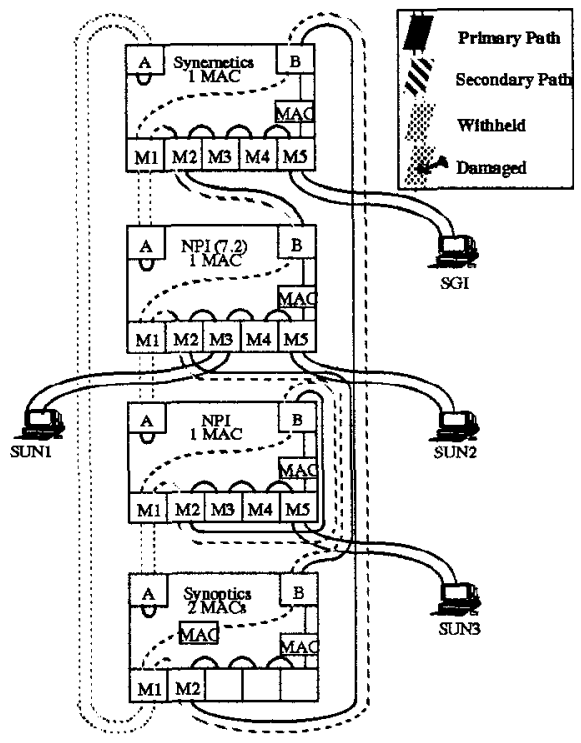

Figure 16: Effective Concentrator Tree with Correct Loopback and damage to a B port Optical Bypass

serted into the data path and when power is removed, the bypass simulates a broken fiber. Figure 17 illustrates this configuration of the bypass unit.

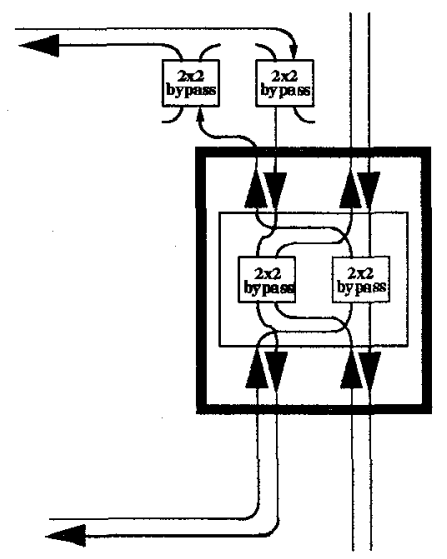

Figure 17: Optical Bypass configuration to disable the a concentrator when power is lost to the bypass

The total optical power loss to the system when the bypass is enabled is twice that of the bypass configuration from Figure 15 for the A/B port side of the concentrator.When the bypasses are not enabled, the effective loss to the network is the same as it was in Figure 15 and there is no additional impact on the network.

Operation of the optical bypasses in Figure 17 is very simple. When power is applied to the unit, the bypasses are engaged and the light is routed from the network to the concentrator ports. When power is removed, light passes from the previous station directly to the next station due to the bypasses in the dark box of Figure 17. The additional switches connected to the B port prevent it from connecting to the $M$ port. With this configuration, the $\mathrm{A}$ and $\mathrm{B}$ ports will only connect to the network and will never form a connection to the $M$ ports.

\section{Conclusions}

Of primary importance to this discussion is the conclusion that the two rules described in section 2.1 are valid for all cases of operation. With this information is it possible to create the concentrator tree with loopback such that the operation is deterministic. In addition, the use of optical bypasses also was verified to have a significant impact on the operation of the network. It was also determined that a simple bypass configuration similar to what is used on the trunk ring of a network is not sufficient to eliminate single points of failure for the stations in the network.

A by-product of the experimentation was the evaluation of some network management products. One obvious conclusion was that none of the existing management software produts could elegantly handle the concentrator tree with loopback. Neither Viewplex nor Optivity were designed to be simple graphical mapping tools, and their function is primarily focused on managing stations in a network. Both Optivity and Viewplex meet their objective.The Silicon Graphics FDDI Visualizer was designed to show the state of the ring but was not capable of displaying the network when the concentrator tree was used.

\section{References}

[1] SAFENET Military Standard, MIL-STD-2204, October 1992.

[2] Ralph, Schellack, Ukrainsky, Weinberg, "Alternate Path Fiber Distributed Data Interface Topology", 17th Conference on Local Computer Networks, Sept. 1992.

[3] Hiles, Marlow, "Approaches for Survivability in FDDI Networks", 17th Conference on Local Computer Networks, Sept. 1992.

[4] Preliminary Draft Proposed American National Standard, FDDI Token Ring Station Management (SMT), ASC X3T9.5, Rev. 7.2, May 1992.

[5] Draft Proposed American national Standard, FDDI Token Ring Station Management (SMT), ASC X3T9.5, Rev 6.2, May 1990.

[6] Case, J.D.; Fedor, M.; Schoffstall, M.L.; Davin, C. "RFC1157: Simple Network management Protocol (SNMP)". May 1990;

[7] SAFENET Military Handbook, MIL-HDBK818-1, October 1992. 